\title{
Mory Diomandé
}

\section{L'altérité de soi et l'esthétique du bruit dans la fulgurance existentielle L'exemple de Manka talèbo de Konan Roger Langui}

\begin{abstract}
Self-Alterity ANd Noise Aesthetic in Existential Fulgurance. The Case Study OF MANKA TALÈBO BY KONAN ROGER LANGUI Abstract: Manka talèbo is a poetic song rooted in the social dynamics, particularly in a libation writing context which is largely based on drum language. The figure of Attoungblan, the speaking drum in the Akan community, is actually at the heart of the work. It has a sacred and sententious voice whose solemn and spasmodic notes have official speech value in the social area. Throughout Roger Langui's work, the speech which is both poetic and initiatory looks toward oneself and the other. Features of an imaginary molded by noise and lightning patterns appears as a clue to a human existence and explores his relationship to a hereafter of Creation.
\end{abstract}

Keywords: Konan Roger Langui; Imaginary;

Alterity, Noise; Poetry; Ritual Song.

\section{MORY DIOMANDÉ}

Université Félix Houphouët-Boigny de Cocody, Abidjan, Côte d'Ivoire diomandemory2002@yahoo.fr

DOI: 10.24193/cechinox.2019.37.15

\section{Introduction}

$1 \mathcal{L}_{\text {de l'Ivoirien Konan Roger Langui. }}^{\text {d'Ino }}$ Parue en 2010, puis rééditée en 2012, dans le sillage de la crise postélectorale qui a secoué son pays, l'œuvre montre toute une " aptitude à la trahison » ${ }^{1}$, c'est-à-dire, selon Robert Escarpit, " une disponibilité telle qu'on peut, sans qu'elle cesse d'être ellemême, lui faire dire dans une autre situation historique autre chose que ce qu'elle a dit de façon manifeste dans sa situation historique originelle $»^{2}$. Cela s'entend dans la mesure où le dire poétique languien s'arc-boute sur l'éternel instinct de préservation de l'homme et sur les particularismes modulant les rapports interindividuels à travers les âges et les sociétés, en dépit des mutations qui peuvent s'y opérer ou que l'espace social peut subir.

Manka talèbo est une expression tirée du baoulé, une langue du Centre de la Côte d'Ivoire. De ce qu'en dit Jérémie Kouadio, linguiste et par ailleurs préfacier du livre, cette expression signifie littéralement «Je suis resté/abandonné sous le mur » ${ }^{3}$. 
L'exégète en fait, par la suite, une lecture contextuelle et symbolique :

Le poète, tel un orphelin, est resté assis sous le mur de la maison familiale en ruine. Orphelin de son terroir natal défiguré par une horde " d'enfants pachydermes » jouant au «Che », dans leurs accoutrements ridicules, il pleure de révolte sur la terre d'Éburnie saccagée par des prédateurs de tout acabit et dénonce avec la force du désespoir, les sempiternelles meurtrissures d'une Afrique qui tarde à se mettre debout ${ }^{4}$.

Ce que nous entendons faire dans le cadre de ce travail, maintenant que le contexte de l'œuvre est circonscrit, c'est de mener une lecture de l'écriture poétique de Roger Langui sur la base de référents d'analyse tirés de l'imaginaire, ayant comme point focal la question de l'altérité. À l'approche de l'œuvre Manka talèbo, on observe, en effet, que l'identité et l'altérité se déploient comme une entité gémellaire dont le point nodal ramène à l'homme dans sa dimension trans-individuelle et groupale. Autant l'identité de l'autre et l'identité collective s'acceptent, autant l'altérité de soi se conçoit à l'aulne des états transitionnels de lêtre.

Sur cette base, l'identité se dévoile comme une donnée dynamique, en même temps que statique. Elle se module suivant le cours de l'existence, se construit et se déconstruit par reniement, adoption ou assimilation d'éléments d'identités extérieurs, convergents ou dissonants, qui viennent se greffer au substrat identitaire du sujet. Et même lorsque l'on dira d'un tiers qu' " il a perdu son identité », ce n'est qu'une autre identité qu'il aura adoptée.
Ainsi, l'homme passe par une série d'étapes de transformation caractérisant son évolution dans le temps, à travers son expérience du monde. Nous tenons cela particulièrement dans les plis de Manka talèbo dont le sous-titre, Chant rituel pour l'Afrique, plonge dans l'initiation d'une parole tambourinée et dans le sacré d'un hymne au souffle vibratoire, à l'image d'une parole primesautière et dépouillée, saisie dans ses premiers jaillissements.

Les quatre étapes desquelles procède le schème initiatique - la mort (symbolique), la renaissance (tout aussi symbolique), le statut nouveau et la réévaluation sociale - deviennent ici symptomatiques d'une situation où l'homme se refuse à une vie d'involuté, une vie qui le révèlerait donc replié sur lui-même et plongé dans une sorte de solipsisme. L'idée de l'expression de soi, de l'acceptation de son identité et de la formulation d'un destin autre, moins anémiant et plus épanouissant, prend du relief. L'approche d'une telle posture s'abouche avec l'idée du bruit, indice des crispations sociales et révélateur d'une présence au monde, dans un univers fulgural tenant à la fois de l'éphémère de la vie, des paramètres de l'imaginaire social et des mèmes qui grèvent l'existence humaine. C'est dans ce champ que s'opère l'embrayage de la parole poétique languienne.

Lordonnance horizontale du texte, qui implique un agencement irréversible des mots, un trajet obligatoire même s'il ne s'agit pas d'un langage proprement logique, se trouve arrêtée, mise en péril : le mot se gonfle par lui-même de significations multiples qui viennent à entraver la marche du discours et, la retardant, la font dévier, 
imposant en contrepoint un cheminement vertical, conférant au texte une épaisseur qu'il n'avait pas d'abord. ${ }^{5}$

Dans ce canevas, les trois types d'écriture poétique que Jean Burgos identifie la révolte, le refus et la ruse ${ }^{6}$ - intéressent, d'autant plus que Manka talèbo se fend d'une écritoire libatoire et d'une parole messianique, annonciatrice de temps nouveaux. Le poète, revêtu du statut de tambourinaire, suit l'exemple d'un personnage théosophique dont la parole médiumnique est à présent opératoire. Avec lui, la poésie devient un chant médium (p. 18) qui, in fine, arrime à l'analyse les manifestations " du désir inconscient et qui facilitent l'investissement de l'énergie psychique [...]). Dans cette visée, les exigences de la censure déclenchent un travail psychique de transformation $»^{7}$ de l'homme et de la société. Précisons qu'ici, la censure est tributaire de l'écart entre les référents symboliques des différents groupes sociaux, l'actualité de leur existence quotidienne et la désarticulation du passé historique des peuples africains. Tout cela conduit à un certain repli sur soi et à l'érection de barrières artificielles, superfétatoires et débilitantes. Celles-ci sont d'abord construites dans le cercle de référence des groupes sociaux, avant de s'exhausser pour contaminer le champ social. Et c'est sur les flancs de ces modalités réflexives, cognitives, conatives et symboliques que se structure l'imaginaire poétique de l'auteur.

\section{Altérité et imaginaire social}

Al'analyse du cadre sociétal qui héberge affleure : "Les universaux [qui] sont des abstracta formés par notre esprit en ne retenant d'un ensemble d'individus que les caractères qu'ils ont en commun ${ }^{8}$. Loin de la Querelle séculaire autour de ce concept, une telle définition, considérée dans son sens immédiat, étire une ligne transversale qui coudoie fortement l'homme, d'abord dans ce qu'il est foncièrement, ensuite suivant le dénominateur qu'il a en partage avec ses semblables. Les traits, disons-le, sui generis qui sont relevés en ce point culminent à hauteur d'un être générique dont l'intérêt n'est pas d'uniformiser ou de styliser une communauté, mais plutôt de souscrire à l'égalité entre les êtres humains, de même qu'aux valeurs humanistes qui s'y adjoignent.

$A$ contrario, le discours social déborde parfois cette notion, se crispant autour de préjugés et de représentations clichéiques qui qui ne font que stigmatiser, exclure et brimer. Ainsi, dans Manka talèbo, le poète se fait véridique, pacificateur et réparateur de torts. L'amorce du processus transformationnel qu'il esquisse, sur la base d'un récit qui s'annonce éclairant, fait porter au texte la charge d'une parole sapientiale, itinérante et révélatrice, une parole au bout de laquelle le parcours destinal de l'homme se révèlera comme une épiphanie.

C'est une histoire

Qui fit germer la terre

Une histoire

À litanies sismiques

Si tu veux l'écouter

Lâche ton avenir

De nègre-thaumaturge

Et si tu veux

Comme moi

Rénover l'histoire 
Porte à fleur de flanc

Le rire sarcastique des labyrinthes

Et tu connaittras

Les chansons des orphelinats

Et le sourire à fève cacaoyère

Mais installe-toi

Sur le prie-Dieu

Car dans le rire sanguinaire

S'installeront

Bientôt les rêves sauvages

Pour saisir à portée de main

Le râle de la paix qu'on passe à tabac (p. 34-35)

L'axe dialectique qui se noue en filigrane du recueil inscrit dans le texte une série de couples antithétiques (la guerre et la paix, les rires et les pleurs, la vie et la mort, le sacré et le profane, le spirituel et le trivial...) dont le dénouement ou le dépassement se réalise dans la pratique ritualisée du verbe. Ainsi, le donné ethnique et les référents totémiques et mythologiques (les attributs religieux, la danse de l'Adjanou, la pratique de la religion bossoniste, les prêtresses komian et autres), la ritualité de la communication orale (à l'occasion de cérémonies de purification, de funérailles, de célébration, etc.), autant que la commission de la parole à réguler la sphère sociale traditionnelle (à travers la parole du père et surtout de la mère, celle du maître, du devin et de Dieu) sont des paramètres de l'imaginaire social akan que Roger Langui réinvestit dans le texte poétique dont le statut de chant rituel reprend certaines caractéristiques des rites de passage.

À ce propos, notons qu'en tant que processus initiatiques, les rites de passage sont vecteurs de transformations, disposant le sujet à faire de l'idée de la conversion une voie cardinale pour transcender sa situation personnelle et réévaluer son statut social, donc sa position face aux autres. En la circonstance, la conversion devra être entendue selon la définition de Denis Thouard, c'est-à-dire comme « l'instant qui césure la vie et inaugure une renaissance spirituelle dans la foi »". C'est d'une foi dans le potentiel de l'homme et dans son ipséité qu'il est question - l'identité nominale ou propre du sujet, qui le particularise - et non dans sa mêmeté. Car, pour rester dans la pensée de Paul Ricœur ${ }^{10}$, la mêmeté trahirait une immuabilité de l'individualité, ce qui détonne quant à la nature dynamique des identités individuelles et de leur rapport à l'altérité.

Sur ces fondements, « la syntaxe de l'Imaginaire $»^{11}$ aiguille l'analyse vers des types d'écriture poétique spécifiques, dont la « conquête. Cette modalité de structuration, rappelons-le, est celle qui répond à une attitude de révolte devant le temps chronologique et la dégradation qu'il implique, révolte qui est à la fois manifestation d'une tendance organique profonde refusant toute finitude et réponse apportée à l'angoisse liée à cette finitude $»^{12}$. Manka talèbo cristallise ce plan de l'expression à travers la figuration d'une parole poétique résolument initiatique. Cette parole, qui ouvre sur les champs fertiles et infinis de la tradition, a pour trépied le vécu historique collectif, l'intercession salvifique des mânes dans le sort des individus et " la voix sacrée de l'Attoungblan [qui] se fait lugubre et funeste pour conter les violences qui ont ensanglanté [...] et qui continuent de balafrer l'Afrique $»^{13}$.

La hantise des chairs meurtries ( $\mathrm{p}$. 18), cramoisies (p. 19) ou suppliciées (p. 26), 
conséquence de la dégénérescence des corps et des actes ineptes de l'homme, au prétexte de domination au long de conflits intercommunautaires, sétiole alors sous les effets reviviscents du Tam-tam rituel (p. 13) pour, enfin, entendre le poète déclarer : $T a$ mort fut une mort tronquée (p. 19). L'idée de la Camarde obturant toute perspective de bonheur et de vie éternelle est ainsi démise sous les injonctions du tambour dont l'onction sacrale est accordée à toutes les victimes d'injustices et de violences sociales. C'est un état de fait dont les reliefs sévaluent plus clairement à l'aulne des velléités de vengeance, désormais conjurées et apaisées. Du coup, les postures victimaires sont abandonnées et le discours social s'en retrouve délesté de toute méchanceté et de toute défiance. L'homme prend conscience du temps qu'il lui reste à vivre et de la nécessité de faire œuvre utile. La dimension phonique du discours joué prend corollairement de l'amplitude.

Écoute donc

Son austère chanson

Qui m’est défi de conscience

Qui m'est songe extatique

Où traînent mélancoliques et mélodieux, Les égouts sauvages et damnés

D’une île en putréfaction!

Écoutez

Écoutez donc chers peuples (p. 13)

C'est suivant cela que la sphère sociale est réglementée, mettant en avant un imaginaire composite dont le tenant principal fait confluer la parole tambourinée, la paix sociale et le bien-être de la communauté, d'autant plus qu'Il est temps de rompre les langages mort-nés/ De ritualiser contre l'emblème maléfique des trônes/À l'arrogance sordide des gales de faubourgs! (p. 17-18). Cet impératif fait prendre aux victimes le statut de martyrs dans la mémoire collective. Du coup, la parole enjouée de l'Attoungblan élit Ces morts/ Dont le glaive invisiblel Soulagera le caur abandonnél Des veuves et des orphelins (p. 21) au panthéon des figures universelles, loin des abysses lointains, des débandades et des rondes cannibalesques (p. 17). Le langage tambouriné se fait ainsi parole présentative, démonstrative et expressive, aussi bien injonctive et (?) exhortative qu'interactive. Le refus de la mort ou de la finitude se greffe de la sorte au parcours existentiel d'un individu à présent résolu à mériter de la vie.

L'idée de la révolte, s'arc-boutant sur l'altérité de soi, prend encore par endroits la forme d'une usurpation de pouvoir, conséquence d'une tendance à étendre son espace vital. Les accotements d'une telle attitude charrient une volonté hégémonique de certains clans mus par le souci du " chez soi ", fait de frictions entre autochtones (soi) et allochtones (l'autre), avec parfois des relents discriminatoires et autarciques. Cette inclination à une hégémonie colonisante se remarque à travers la détestation de l'étranger-celui qui n'est pas (comme) nous -, l'accaparement des biens d'autrui et/ou le pancartage de son territoire avec des slogans xénophobes, ivoiritaires ou nationalitaires; attitudes que le poète s'évertue à annihiler.

La révolte s'appréhende, en sus, dans le sein d'une surenchère de l'identité au nom de laquelle des ethnies se déclarent meilleures que les autres ou supérieures à elles. Elle se traduit également par un déni de la réalité et une déresponsabilisation (l'on se dédouane, s'innocente de toute 
part dans les conflagrations sociales, pour ensuite culpabiliser l'autre). Et c'est à la lumière de l'histoire et du contexte social ivoirien, dont se nourrit l'œuvre, que la présente analyse émarge.

Au final, l'altérité se transmue en une altération de l'identité, celle de soi bonifiée et celle de l'autre dévalorisée. Par contrecoup, les idées-forces sont bousculées, segmentées selon d'autres référents qui ne sont aucunement adoubés par la réelle configuration de la société. Ces nouvelles subdivisions, artificielles, relèvent davantage de manipulations politiciennes et de considérations sectaires, motivées par une altérité clanique et tournée vers l'intérieur. C'est pour exorciser cette crise des valeurs et du véritable que Roger Langui mobilise la parole tambourinée. Suivant ses inflexions et sous sa férule, "tout se passe comme si la parole qui jaillit sous l'effet des forces souterraines, par une sorte de mouvement dialectique, devenait agissante à son tour $»^{14}$, dans le sens du salut de la société.

Lidée de la révolte est complétée par des schèmes illustrant le refus. Ceux-ci s'appuient sur la brisance des mots dont le poète use, c'est-à-dire sur la portée sémique ou symbolique des mots à charge dialectique, induisant l'adversité et traduisant la capacité à reformer l'existant et à casser toute résistance.

Je revendique

Des mots sur civière $[\ldots]$

Des mots fouets et hameçons

Des mots enfourchés à califourchon de la Haine...

Des mots

Points d'orages et de tourbillons

Des mots tomahawks [...]

Des mots clés
Qui résonnent

Des mots rassemblés en colonies du refus $[\ldots]$

Ces mots brasiers enturbannés de cendre (p. 27-28)

Civière, fouets, hameçons, enfourchés, orages, tourbillons, tomahawks, refus, brasiers, cendre... sont autant de lexies qui témoignent d'antagonismes, d'une instabilité des lieux et de l'ambition de l'auteur de désinstaller l'existant, dans un espace qui, symboliquement, est vécu comme un autre soi-même. Lespace est, en effet, une part profonde de nous, en ce qu'il rattache et attache à un lieu spécifique, englobant, dans un double rapport de possession (nous possédons l'espace, autant qu'il nous possède). Cet enracinement charrie au passage tout un ensemble d'artefacts porteurs de significations sur les origines, l'éducation, les croyances ou la langue d'un individu, de même que son rapport aux autres.

Par ailleurs, l'écriture du refus procède singulièrement d'une « tendance profonde de refus devant le temps chronologique $»^{15}$ :

La réponse à l'angoisse devant la temporalité, à la conscience de toute finitude, n'est plus spontanément cherchée, cette fois, dans la mainmise sur l'espace et son remplissement, mais au contraire dans la délimitation d'espaces privilégiés où se mettre à l'abri du temps. Ainsi le schéma directeur de cette syntaxe va-t-il être d'approche, de construction, de renforcement de refuges toujours menacés, toujours à restaurer en des espaces tendant à se réduire, comme s'il devait finalement se trouver un lieu clos - oublié - où échapper au temps. ${ }^{16}$ 
La conséquence immédiate d'une telle posture est la représentation ectoplasmique ou spectrale de la vie sociale dont l'échec est tantôt prononcé par le poète. Le regard que celui-ci porte sur une Afrique mortifère est constamment embué, à force de déceptions et de désolations. C'est naturellement dans ce champ qu'officie le tamtam parleur, témoin oculaire et auriculaire de son temps. Son chant lessiviel, parce que purificateur pour la société, revendique DES MOTS DE TORTURES INFINIES (p. 29), une parole résolument engagée au bénéfice de l'Homme.

Sous la sangle des mains molles

Dans le souffle agonisant des spectres [...]

Gisant comme des lambeaux de chairs Sur la route des obus

À la racine des crucifix abandonnés (p. 27)

De ces figurations d'une vie calcinée, transparaît la ghettoïsation de certaines communautés, en même temps que le poids des poncifs ou des a priori. Le reflet des existences cataloguées, comprimées, dévastées, voire décimées, induit le retour à un passé heureux, temps des félicités perdues. Les lieux confinés et intimes, que sont le cœur, la conscience et les rêves, deviennent les espaces privilégiés que l'homme investit pour se défaire des sourires froissés (p. 17) et pour s'accorder un moment de répit.

Tout cela, in fine, et à travers l'exercice ritualisé de la parole tambourinée, augure de la métamorphose des individus qui non seulement se dévoilent à eux-mêmes et en eux-mêmes, mais se découvrent également à leur alter ego, cet autre dont l'image spéculaire est révélatrice du soi profond. Cette opération transformative s'origine dans la déception qu'inspire l'existence. Toutes les vies envoyées ad patres, du fait de conflits sociaux inhérents à la décrépitude morale et à la rapacité ambiante, sur fond de phobie de l'autre, Roger Langui espère leur absolution dans le giron de Koumoin si, le Pays des ancêtres (p. 46), l'ailleurs lointain, oublié, où l'on se réconcilie avec soi-même, par ses racines, ses origines. Car à la vérité, la haine de l'autre est symptomatique d'une haine de soi-même, donc le signe d'une défaillance de la société dans ses fondements et dans son fonctionnement. Les manifestations d'un tel processus, plurielles et éclatées, empruntent les sillons d'une thérapie collective, par le truchement des battements tambourinaires réunissant le peuple dans toute sa diversité.

\section{De l'imaginaire et du statut de la parole tambourinée}

T a parole qui sourd des manifestations Lritualisées est symboliquement portée par la voix expiatoire et propitiatoire de l'Attoungblan, le tam-tam parleur dont le langage, tambouriné, ramène à l'origine du monde. En effet, de ce que dit le drummologue Niangoran Bouah sur les fondements théoriques de ce membranophone, le jeu du tambour parleur débute comme suit :

Le Tambour dit : "Au commencement, Dieu a créé Sen. Sen, leénergie qui donne la vibration, la vibration qui donne le son et la multiplication des sons qui donne la parole. Dieu a créé le tambour qui est le symbole naturel de la parole et le réceptacle de la parole primordiale de Dieu ». Voilà 
la première parole du tambour, qui continue ainsi : «Dieu, en créant son monde, qu'avait-il créé en tout premier lieu ? Dieu, en créant son monde, a créé Sen et a créé le tambour ». Puis on invoque lesprit qui vient animer le tambour. Le tambour est en effet à la fois objet naturel, matière et esprit qui l'anime $^{17}$.

Le tambour se fait ainsi l'égal du Verbe créateur, dans la Bible. Dans la tradition prophétique de l'Islam il est le pendant du Calame, l'équivalent de la plume à écrire, un objet auquel le Dieu Créateur aurait enjoint de transcrire le devenir de l'Univers. Au fur et à mesure donc des paroles divines, se formaient des graphies, et au fil de ces graphies, se construisait un monde, naissait le monde, advenait la vie. Par-là même, le monde co-naissait avec le Verbe ou l'Écriture. L'un dans l'autre, il y a, à la genèse du monde, la parole fécondante.

Le trait cosmologique susdit draine un arrière-plan métaphysique et symbolique dont se saisit Roger Langui pour faire basculer la société humaine dans une phase réflexive. Il en est ainsi car, par le tambour, la société est psychologisée, ramenée à son avènement et à ses tâtonnements, comme un enfant au stade du miroir lacanien, revisitant symboliquement l'inconscient collectif au prix de mille pulsions scopiques, dont le point d'arrimage vient se fixer dans l'idée du regard : regarder et être regardé. Par conséquent, il se joue ici un va-et-vient constant entre l'identité et l'altérité, entre regard intérieur (introspection, image de soi), regard extérieur porté (jugement de valeur ou de fait) et regard extérieur subi (préjugés, poncifs). À travers de telles structures mentales, l'homme est censé redécouvrir sa nature profonde, apprendre de sa vie passée et réapprendre à aimer son reflet. C'est l'aube d'une ouverture de soi et à soi, comme une thérapie primale, qui poserait donc une phase de remémoration, permettant au sujet de revivre son passé et d'accepter plus facilement ses fautes et sa situation.

Il en ressort, par ailleurs, que l'homme est devenu étranger à lui-même, à force d'adversité, d'individualisme et d'ambitions nombrilistes. Dans un tel contexte, fait de défiance dans les rapports interpersonnels, et du fait de l'inconfiance qui mine les individus, l'identité se structure dans le reflet de l'altérité. Il s'agit d'une identité tentaculaire où la haine précieuse (p. 14), la haine envers l'autre, qui colle tant à l'âme, est tributaire de facteurs intrinsèques se rapportant au sujet concerné (son idiosyncrasie et son instinct de préservation, par exemple) ou extrinsèques, notamment les manipulations politiciennes et les stratifications sociales qui excluent. S'y ajoute la présence de l'autre vécu comme différent, comme étranger, parce que nétant pas nous, et aussi perçu comme une menace, d'où le glissement lent et insidieux vers une xénophobie, artificiellement conçue et distillée, dirigée à la fois vers l'intérieur (ses propres concitoyens, d'ethnies différentes) et l'extérieur du pays (les expatriés, les immigrés), et modulée par le cadre de référence des individus. Ce sont là des éléments qui éclairent le cadre conflictuel de l'œuvre Manka talèbo.

Cette part obscure de l'homme que Roger Langui met à l'index fait correspondre l'altérité de soi avec l'autre nousmêmes, le côté lumineux ou obscur de soi, selon les circonstances. En référant au contexte d'émergence du livre, et dans la 
perspective de la réconciliation nationale à laquelle le poète souscrit, l'altérité de soi incline à panser en nous ce soi blessé, la part victimisée ou auto-victimisée de soi. À force de frustrations, de brimades et d'injustices, la population se retrouve rétive au pardon et à l'oubli, encore moins à la résipiscence. La vendetta écumante de sang, dans les flots de sang (p. 19), au pays des crimes (p. 26) ou encore, au " pays du sang ${ }^{18}$, pour emprunter à la formule d'Yves Bonnefoy, est suscitée par des larmes mortifères de Guitrozon/Des charniers délinqués de la voûte de nuits (p.16) et des colonies étranglées/ Exsangues, gisant dans la marre éternelle des pensées [...], pour une république sanglante qui s'empiffre de viol (p. 17).

Face à cela, battre le tam-tam devient un acte de repentance et de pénitence. L'on comprend alors pourquoi le poète incite à battre frénétiquement et tous azimuts :

Battre des mains et des pieds

Battre à pas feutrés le rythme de mon peuple

Battre du cœur

De la poitrine le tam-tam sonore de la tribulation!»

Regardez battre

L'anneau irrésistible

Des tam-tams névralgiques

De mes mains endolories (p. 25)

La ruse, en tant que forme d'écriture poétique, s'ajuste sensiblement au réalisme qui caractérise la parole prodigieuse de l'Attoungblan. Pour le poète, maître diseur de vérités éternelles officiant en ces lieux, ce procédé doit être lu sur les flancs du progrès. « Cette modalité de structuration correspond pour sa part à une attitude radicalement différente des deux précédentes, puisqu'elle ne dit pas non au temps et ne lui oppose ni révolte ni refus. Tout à l'inverse, elle représente une attitude positive qui est, ou plutôt semble être, acceptation de l'inéluctable déroulement chronologique ${ }^{19}$. Et si le poème se fait chant médium (p. 18), c'est bien pour coller à l'asphalte de cette figuration cognitive et imaginaire. La parole de tam-tam (p. 22) qui tonne alors, portée par l'épique souffle millénaire (p. 29), se mue en héraut de mon peuple (p. 21).

Des référents mythologiques viennent s'y adjoindre : le redouté Ko'ngo, l'enjambeur de fleuve (p. 30), la figure mythique de la reine Pokou, Attiégouale Dieu de l'hospitalité et de la justice chez les Baoulé (p. 41), les Féwa (p. 44) désignant les trois premiers morts d'une famille (akan) et dont l'esprit est protecteur pour le reste de la fratrie. Une référence est même faite à Pégase (p. 28), le mythique cheval ailé. Ces différents paramètres de l'imaginaire languien aident à nouer un espace conjonctif au sein duquel les écarts sont corroyés et dépassés. Au cumul, le tambour, qui rythme toute la communion sociale, devient l'adjuvant de la figure démiurgique du poète, condensant tous ses mécomptes et toutes ses aspirations.

Danse!

Danse à l'écoute de ma voix

À l'écoute de la longue mimique de ma voix

Danse au son

De ma PAROLE

De ma voix

Ma voix de dix mille poètes

Enterrés

Incarnés

Incarcérés

Incinérés (p. 33) 
Suivant une structure scalaire descendante, le tam-tam parleur se fait d'abord la voix de Dieu, ensuite symboliquement celle du chef, puis du peuple, enfin de l'individu, voire du marginal qui se l'approprie. Quant à l'homme, il se résout à accepter son destin avec dignité et en toute responsabilité, conscient du fait que, quelle que soit la gravité des actes posés, rien n'est irrémissible.

\section{De la fulgurance et du bruit comme indice du moi et son double}

$\mathrm{I}^{1}$ existe une relation intime entre les deux types d'écriture poétique que sont la révolte et le refus. Dans ce canevas, les conflits sont toujours opposition entre soi et l'autre, mais aussi entre soi et un soi autre, cohabitant dans l'intimité de la conscience. Aussi, exercer une violence sur autrui, c'est, dans un jeu de substitution-projection, vouloir s'approprier son corps et sa vie. C'est chercher à les posséder, à les soumettre, pour en disposer à sa guise, ne serait-ce que le temps d'un accès de colère ou d'une vengeance.

Suivant le choc des identités nominales qui en résulte, Manka talèbo se structure au gré d'un schéma énonciatif à double levier, où la prise de parole s'incarne dans le tam-tam parleur, avec d'abord la parole humaine confiée à l'instrument, ensuite la parole tambourinée décryptée par le tambourinaire, puis portée au peuple. Tous les éléments constitutifs du schéma communicationnel standard transparaissent en l'espèce, se crispant autour d'un émetteur et d'un récepteur, d'un message, d'un canal et d'un codage/décodage, ainsi que d'une rétroaction, d'un référent et des bruits.

Sur cette dernière composante, notons que si le bruit détonne dans la vie quotidienne et indispose, dans le cas présent, il ramène plutôt à tout élément pouvant perturber la transmission et le bon déroulement d'une communication. Ici donc, les bruits seront faits des ressentiments, de la colère et de la position victimaire de certains suppliciés des conflits sociaux. Ils tiennent de la douleur éprouvée par tous ceux qui, jusque-là, n'ont pu faire le deuil d'un être cher ou des biens dont ils ont été spoliés, de même que du besoin de justice et de réparation. Le bruit sera donc tout ce qui vient brouiller les signaux de la réconciliation nationale et la disposition des individus à pardonner. C'est au bout de cette approche de l'autre - l'autre, ce soi nouveau resulté d'une réconciliation nationale portée frénétiquement par la parole tambourinée, d'une part, et l'autre, autrui, notre alter ego, d'autre part - dans le dessein de réaliser l'épiphanie de l'autre nous-même, jusque-là égaré dans le tourbillon des rancœurs et de la division, que les rapports sociaux, c'est-à-dire les relations à l'autre, seront modifiés et bonifiés.

En outre, le bruit, parce qu'il est le résultat d'une action, ramènera à tout ce qui crée en l'homme un ressenti, une réaction. C'est ce qui émeut, c'est qui échappe à la linéarité de la vie et trouble le cours monocorde. C'est tout ce qui résonne à l'oreille, à nos sens, jusqu'à l'âme. Parce que le silence s'entend, il revêt également les attributs du bruit, qui se fait tantôt l'indice d'une présence, d'une absence ou d'une non-présence, tantôt le pendant du mutisme, du bâillonnement ou de l'indifférence, dans un univers basculant tantôt dans un hors-temps. Suivant cela, les structures de l'imaginaire languien se refusent à toute mesure linéique, pour faire de l'altérité un face-à-soi dont l'idée alinéaire 
serait la configuration d'un univers symbiotique où les images archétypales, dans un effet de dédoublement, seraient reformées ou démises. Comme le dit le poète,

Rarement, te dis-je

Ils trouveront la juste cadence

De ma passion africaine

Et de tous les Mao trahis

De tous les Che qu'on éventre

De tous les héros qu'on désaltère

À coups de sang et de bile (p. 35)

Par ailleurs, la prise de parole est faite par un $j e$ individuel parlant en son nom propre, puis par un $j e$ d'association et d'identification s'accommodant du possessif - ma terre, mon sang, mon peuple d'otages (p. 26) -, enfin par un nous inclusif : nos colères infructueuses, notre combat, notre terre, nos enfants, etc. " Les jeux verbaux si vertigineux qui peuvent résulter d'une telle pratique $»^{20}$ relèvent d'une prise de parole éclatée et multiforme qui condense le vécu individuel, les aspirations et les revendications collectives.

Ainsi, le tambour parleur, cet instrument de percussion, loin dêtre un simple objet autotélique, fait écho à une représentation dialectique de l'espace social, à travers le lien entre l'individu et le groupe, même entre le visible et l'invisible. Cette configuration duale est présente dans le champ traditionnel akan que le poète exploite notamment. En cette altérité extérieure, constituée par l'alentour, à la fois par le lieu où l'on se tient et par l'endroit où l'on n'est pas, s'incarne la part double de l'homme dont les formes sont déclinables à souhait : gentil/méchant, dominant/dominé, vivant/mort, damnélélu, bon/mauvais, etc., toujours dans un rapport à l'autre.
La posture du refus se justifie encore par la relation avec autrui, ce besoin de porter la voix de l'altérité, ici motivée par le rattachement et l'attachement à un lieu refuge.

Mon peuple qui ne rit pas

Qui gémit

Qui n'agit pas

Qui vagit

Qui ne rugit pas

Qui s'agite

Qui ne vit pas

Qui gît (p. 20)

C'est en ce peuple sien, dont l'omniprésence est campée par le traitement anaphorique du pronom qui (huit occurrences dans l'extrait ci-dessus), que le poète se reconnaît. Et c'est à travers cet autre lui-même, la quintessence de son être, que l'identité individuelle se structure et s'établit. Parler de l'autre revient alors à parler de soi, car c'est par rapport à son proche que l'on se détermine, par identification, assimilation ou dissociation. Le tam-tam parleur, générateur de danse, voire de transe délivrante, dévoile « un poème expiatoire, un chant rituel de libations $»^{21}$, où l'homme se découvre soit en position d'orant (disposé à la prière et à faire amende honorable), soit dans une posture auto-flagellante (pour un mea culpa ou une autocritique). Puisque le poème est un témoignage sur une période clivée de l'histoire de pays en crise, Manka talèbo est un chant exutoire et testimonial, une parole ritualisée dans un univers ramenant aux funérailles des morts qui vivent ( $\mathrm{p}$. 21).

Dans les pas de cette écriture libatoire, au son de l'Attoungblan, la fulgurance existentielle se décline. Celle-ci se caractérise 
d'entrée par l'évanescence de la vie et par la recherche obsédée et obsédante d'une exposition sociale qui trahit la submersion de l'identité et de l'altérité dans la vie moderne. La fulgurance existentielle tient encore de l'éphémère des relations humaines, qui se font et se défont au gré des humeurs et des intérêts du moment, de la volatilité de la parole à laquelle presque personne ne croit, et à la vacuité du temps qui passe, qui module l'existence et qui fait de tout une urgence ou un besoin.

En outre, la fulgurance est marquée par le motif de la blessure qui devient le symbole manichéen de l'adversité, de la faiblesse et de la bravade, le signe de l'épreuve, du sacrifice et de la salvation. Tout un imaginaire fait de douleurs et de violences se structure ainsi dans Manka talèbo autour de la blessure. À celle infligée au corps, s'adjoint celle faite au cœur, à l'esprit et à l'âme, à autrui, à la nature et à Dieu. Toutefois, le sang qui s'écoule à l'intérieur et à l'extérieur du corps transmue la blessure en un fait de connaissance de soi et de co-nnaissance (à l'autre). Lon se révèle à soi, dans sa capacité de résilience, l'on apprend, par la douleur, le prix de la vie. Par conséquent, l'infinitésimalité ou l'insignifiance de l'existence humaine à l'échelle du monde s'apprécie amplement. Roger Langui rejoint ainsi Alain Jouffroy qui déclare ceci : "Pour le poète, le monde, le sang et la pensée ne font qu'un. Être dans la pensée, ceest saigner dans le monde $»^{22}$. Cette consubstantialité reprend les traits assimilatifs du même et du divers, du soi et de l'autre ou encore de l'identité et de l'altérité.

Parce que la blessure est infligée ou auto-infligée, elle induit un rapport à l'autre ou à soi. Les crises ivoiriennes successives, depuis la boîte pandorique ouverte par le putsch de 1999, servent de terreau primaire à l'œuvre Manka talèbo dont le long souffle s'accommoderait d'une étude mythanalytique, laquelle, comme le spécifie Denis de Rougemont, viserait à « élucider les motifs de nos choix et leurs implications trop souvent inconscientes, spirituelles autant que sociales $»^{23}$.

Dans le prolongement de l'œuvre, nous constatons une duplication des voix dans le texte, d'abord par la présentation de certains passages en italique ou dans une graphie normale, ensuite par les guillemets français en régime de citation et, enfin, par les guillemets anglais pour l'intercalation d'une autre pensée dans le texte. Symboliquement, à travers cette sorte de dialogue différé ou par procuration, il y a une mise en écho des consciences. Cette compilation de voix génère naturellement une compilation de visages lesquels, dans l'imaginaire languien, fonctionnent comme des espaces.

Autant « chambre et maison sont des diagrammes de psychologie qui guident les écrivains et les poètes dans l'analyse de l'intimité "24, comme le spécifie Gaston Bachelard dans La poétique de l'espace, autant les visages sont pour Roger Langui un lieu de vie, une matière, une forme ou des formes, un masque, une épreuve vécue et racontée, une âme incarnée ou trahie. Chacun de ces visages-espaces qui défilent racontent donc une histoire. Et ces visages individuels donnent in fine une combinatoire de visages dont la conjonction des histoires ramène à la vie dans ce quelle a de plus immédiat et de plus profond : la présence de l'autre.

Par ailleurs, en raison de son caractère sacré et en tant qu'objet cultuel et festif, le tam-tam parleur émarge doublement aux régimes diurne et nocturne, avec des 
structures mystiques dont le schème et les relevés isotopiques pourraient être étudiés. L'esthétique du bruit qui s'y greffe, en plus de trahir une présence, peut faire prendre à cette notion la forme du deuil ou d'un éclat de sourire, d'un déséquilibre ou d'une marche, de l'espoir ou d'une danse, bref, de toute chose qui embellisse l'existence, au sens de l'animer, de lui donner corps, vie et sens. En effet, le langage tambouriné exploité par l'auteur révèle la faillite du langage social usuel, qui est trop souvent la cause de vexations, de crispations et de tensions sociales.

Dans le cas spécifique de l'écriture poétique de la ruse à laquelle Roger Langui recourt, le procédé est employé pour déjouer le cours des choses. Toute la négativité, toutes les souffrances accumulées et compressées dans la psyché des individus sont à présent symboliquement sublimées dans la voix tonale du tambour. Du coup, la pratique musicale et poétique cristallise les désirs latents et profonds de l'homme et permet de transformer toute les charges émotionnelles négatives en forces créatrices. Le processus sublimatoire freudien aboutit à des actions " orientées vers des buts socialement supérieurs $»^{25}$.

La démarche zététique que le poète adopte corollairement, en questionnant les problématiques sociales et en recherchant des explications objectives ou rationnelles aux phénomènes qui surviennent, aide à saisir la portée de son engagement. Comme l'indique Ilona Kovács, « la reconnaissance de l'image spéculaire implique un clivage entre le moi et l'autre, entre sujet et objet en ce que cette image est comme un autre : la reconnaissance de soi s'accompagne donc d'une méconnaissance quant à la vérité de l'être ${ }^{26}$. C'est une problématique dont la résolution transite par l'appréhension du lien insécable faisant de l'altérité le véritable pendant de l'identité.

\section{Conclusion}

T 'un des enjeux de la présente étude fut Lde « montrer comment le texte [...] peut être parlé mais aussi révélé, peut être actualisé et vécu dans ses potentialités, quel que soit l'Imaginaire de son écriture, mais à condition d'en savoir déchiffrer la syntaxe $»^{27}$. Il en ressort que Manka talèbo est un chant hymnique dont le caractère sacré et la portée sociale lui conferrent un statut unique dans la communauté. $\mathrm{Si}$, comme l'indique Niangoran-Bouah, « les ancêtres utilisèrent le tambour parleur pour communiquer avec les dieux, avec l'esprit des ancêtres et enfin pour dialoguer avec les divinités lors d'une cérémonie rituelle ${ }^{28}$, la nouvelle donne, en plus de reconduire cela, enjoint davantage la parole rythmée à la pacification des cœurs et à l'apaisement du front social. Le tambour parleur est ainsi commis à dire la parole régulatrice, celle-là qui préside à l'avènement d'une société apaisée et équitable, où l'enfant maudit des prés (p. 18) pourra rêver de se voir un jour prince adulé.

L'Attoungblan ce jour-là a chanté à tue-tête à tue-corps à tue-rêve (p. 13)

"Lutilisation métaphorique et métaphysique des objets parlants $»^{29}$, en l'occurrence l'Attoungblan, élève le discours poétique à un degré qui côtoie l'anagogique, avec des mots pénétrants qui ne seront décryptés que par l'œil de l'initié. 
Dans cet ordre d'idées, les rapports entre identité et altérité aident à construire l'individu nouveau attendu au sortir du processus de paix. Il en est ainsi car l'idée du temps aliénant et désarmant est comblée par la volonté du poète de devenir un être autre, nouveau, au contact de l'autre.

En définitive, nous notons que Manka talèbo figure un imaginaire dense, lequel, à l'instar d'un divan de "psy ", autorise une thérapie profonde, salvatrice pour tous. C'est la raison pour laquelle la démarche que nous empruntons à Jean Burgos « amorce un retour à la création linguistique, une nécessaire réconciliation de la langue et de l'imaginaire $"^{30}$, pour peu que l'homme veuille s'armer d'idées et de courage. Dans une société ivoirienne qui compte soixanteet-trois ethnies, et par-delà, dans une société humaine où le goût de soi rend les uns et les autres parfois allergiques à la présence d'autrui, l'altérité est ici exploitée par Konan Roger Langui comme une occasion, non pas d'exemplification du quant-à-soi, mais d'un face à face avec soi, où l'autre sert de regard, où l'autre, c'est aussi soi-même.

\section{RÉFÉRENCES BIBLIOGRAPHIQUES}

Bachelard, Gaston, L'eau et les rềves. Essai sur l'imagination de la matière, Paris, Librairie José Corti, 1942, 2005

Bachelard, Gaston, La poétique de l'espace, Paris, Les Presses Universitaires de France, $3^{\mathrm{e}}$ édition, 1961 Bonnefoy, Yves, Anti-Platon, Paris, Gallimard, 1970

Bricka, Blandine, Faudet, Georges, « Niangoran-Bouah, Père fondateur de la Drummologie, in L'arbre à Palabres no.12, Novembre 2002, p. 108-116

Burgos, Jean, Pour une poétique de l'Imaginaire, Paris, Éditions du Seuil, 1982

Chelebourg, Christian, L'imaginaire littéraire. Des archétypes à la poétique du sujet, Paris, Nathan, 2000

Chelebourg, Christian, L'imaginaire littéraire. Des archétypes à la poétique du sujet, Paris, Nathan, 2000

Dyens, Olivier, La Condition inhumaine. Essai sur l'effroi technologique, Paris, Flammarion, 2008

Freud, Sigmund, Introduction à la psychanalyse, Paris, Éditions Payot, 1962

Hubert, Etienne-Alain, Bernier, Philippe, André Breton, 1997, Disponible sur : http://culturesfrance. com/adpf-publi/folio/breton/index.html.>

Jakobson, Roman, Huit questions de poétique, Paris, Éditions du Seuil, 1977

Jouffroy, Alain, Préface de Clair de terre, Breton (André), Paris, Edition Gallimard, 1966

Kacou, Vincent Davy, L’herméneutique du soi chez Paul Ricceur, Prolégomènes à une éthique de la Reconstruction de l'Afrique, Paris, Mon Petit Editeur, 2014

Kaës, René, Les théories psychanalytiques du groupe, Paris, PUF, 1999

Kouadio, N'guettia Martin, Poétique de l'imaginaire et construction du sens. Schèmes, images, syntaxe et signifiance, France, Université de Savoie, 2010

Kovács, Ilona, Introduction aux méthodes des études littéraires, Budapest, Bölcsész Konzorcium, 2006

Langui, Konan Roger, Manka talèbo. Ou chant rituel pour l'Afrique, Paris, Publibook, 2012

Niangoran-Bouah, Georges, « Symboles institutionnels chez les Akan », in L'Homme, Études d'Anthropologie Politique, tome 13, no. 1, Février 1973, p. 207-232, Disponible sur : http://www.persee.fr/doc/ hom_0439-4216_1973_num_13_1_367334

Obou, Louis, "L'univers des objets parlants dans la culture africaine : le Masque et le tambour », in NAC's Journal of African Cultures E'Civilizations, no. 1, 2015, Paris, New African Cultures, Disponible sur : http://nofi.fr/nofipedia/6137

Ricœur, Paul, Soi-même comme un autre, Paris, Seuil, 1990

Rougemont, Denis de, Les Mythes de l'amour, Paris, Albin Michel, 1996

Rougier, Louis, Histoire d'une faillite philosophique : la Scolastique, Paris, Jean-Jacques Pauvert, 1966 
Théorie de la littérature (1975-1985 et 1992-1997), Octobre 2008, Disponible sur : http://www.scribd. $\mathrm{com} / \mathrm{com} / \mathrm{doc} / 15723977 /$ THEORIE-DE-LA-LITTERATURE〉.

Thouard, Dénis, Stylistique herméneutique :J. G. Hamann, 2003, Disponible sur : http://revue-texto.net/ lettre/Thouard_Hamann.html.

\section{Notes}

1. Robert Escarpit, Théorie de la littérature (1975-1985 et 1992-1997), Octobre 2008, Disponible sur : http://www.scribd.com/com/doc/15723977/THEORIE-DE-LA-LITTERATURE〉.

2. Ibid.

3. Jérémie Kouadio, Préface de Manka talèbo. Ou chant rituel pour l'Afrique, Paris, Publibook, 2012, p. 9.

4. Ibid. p. 9-10.

5. Jean Burgos, Pour une poétique de l'Imaginaire, Paris, Éditions du Seuil, 1982, p. 10.

6. Ibid., p. 155-169.

7. René Kaës, Les théories psychanalytiques du groupe, Paris, PUF, 1999, p. 74.

8. Louis Rougier, Histoire d'une faillite philosophique : la Scolastique, Paris, Jean-Jacques Pauvert, 1966.

9. Dénis Thouard, «Stylistique herméneutique : J. G. Hamann », 2003, Disponible sur : http://revuetexto.net/lettre/Thouard_Hamann.html.

10. Paul Ricœur, Soi-même comme un autre, Paris, Seuil, 1990.

11. Burgos, Pour une poétique de l'Imaginaire, p. 155.

12. Ibid., p. 156-157.

13. Kouadio, Préface de Manka talèbo, p. 10.

14. Marguerite Bonnet citée par Etienne-Alain Hubert, Philippe Bernier, André Breton, 1997, Disponible sur : ‘http://culturesfrance.com/adpf-publi/folio/breton/index.html.».

15. Burgos, Pour une poétique de l'Imaginaire, p. 160.

16. Ibid., p. 160.

17. Blandine Bricka, Georges Faudet, « Niangoran-Bouah, Père fondateur de la Drummologie », in L'arbre à Palabres no. 12, Novembre 2002, p. 113-114.

18. Yves Bonnefoy, Anti-Platon, Paris, Gallimard, 1970, p. 13.

19. Burgos, Pour une poétique de l'Imaginaire, p. 165.

20. Ibid., p. 156.

21. Kouadio, Préface de Manka talèbo, p. 11.

22. Alain Jouffroy, Préface de Clair de terre d'André Breton, Paris, Gallimard, 1966, p. 15.

23. Denis de Rougemont, Les Mythes de l'amour, Paris, Albin Michel, 1996, p. 43.

24. Gaston Bachelard, La poétique de l'espace, Paris, Les Presses Universitaires de France, 3 édition, 1961, p. 64.

25. Sigmund Freud, Introduction à la psychanalyse, Paris, Éditions Payot, 1962, p. 15.

26. Ilona Kovács, Introduction aux méthodes des études littéraires, Budapest, Bölcsész Konzorcium, 2006, p. 93.

27. Burgos, Pour une poétique de l'Imaginaire, p. 156.

28. Georges Niangoran-Bouah, «Symboles institutionnels chez les Akan », in L'Homme, Études d'Anthropologie Politique, tome 13, no. 1, Février 1973, p. 229-230, Disponible sur : http://www.persee.fr/ doc/hom_0439-4216_1973_num_13_1_367334.

29. Louis Obou, «Lunivers des objets parlants dans la culture africaine : le Masque et le tambour », in NAC's Journal of African Cultures E Civilizations, no. 1, 2015, Paris, New African Cultures, Disponible sur : http://nofi.fr/nofipedia/6137.

30. Christian Chelebourg, L’imaginaire littéraire. Des archétypes à la poétique du sujet, Paris, Nathan, 2000, p. 77. 